\title{
tic\&société
}

Vol. 13, No 1-2 | 1er semestre 2019 - 2ème semestre 2019

Les industries culturelles à la conquête des

plateformes?

\section{Filière de l'audiovisuel et plateformes SVOD : une analyse croisée des stratégies de Disney et Netflix}

\section{Lucien PERTICOZ}

\section{OpenEdition}

\section{Journals}

Édition électronique

URL : http://journals.openedition.org/ticetsociete/3470

DOI : 10.4000/ticetsociete. 3470

Éditeur

Association ARTIC

Édition imprimée

Pagination : 323-353

\section{Référence électronique}

Lucien PERTICOZ, "Filière de l'audiovisuel et plateformes SVOD : une analyse croisée des stratégies de Disney et Netflix », tic\&société [En ligne], Vol. 13, № 1-2 I 1er semestre 2019 - 2ème semestre 2019 mis en ligne le 20 avril 2019, consulté le 10 décembre 2020. URL : http://journals.openedition.org/ ticetsociete/3470 ; DOI : https://doi.org/10.4000/ticetsociete.3470 
tic\&société - 13(1-2), 2019

\section{Filière de l'audiovisuel et plateformes SVOD : une analyse croisée des stratégies de Disney et Netflix}

\section{Lucien PERTICOZ}

Lucien Perticoz est enseignant-chercheur en sciences de l'information et de la communication à l'université Jean Moulin Lyon 3 et membre de l'équipe pluridisciplinaire MARGE (E.A. 3712). Ses travaux se positionnent au croisement de l'étude des évolutions des pratiques culturelles et d'une approche critique des mutations récentes des industries de la culture et des médias. II a dirigé, avec Jacob Matthews, l'ouvrage collectif L'industrie musicale à l'aube $d u X X I^{e}$ siècle. Approches critiques (2012). lucien.perticoz@univ-lyon3.fr 
Filière de l'audiovisuel et plateformes SVOD : une analyse croisée des stratégies de Disney et Netflix

\title{
Filière de l'audiovisuel et plateformes SVOD : une analyse croisée des stratégies de Disney et Netflix
}

\begin{abstract}
Résumé : Notre contribution se focalisera sur les stratégies financières et éditoriales mises en œuvre par Disney et Netflix afin de mieux cerner les logiques des mutations qui traversent le marché de l'audiovisuel. L'enjeu de cette analyse est donc d'appréhender les formes d'ajustements réciproques qui s'opèrent entre le marché des plateformes SVOD et celui des médias audiovisuels "traditionnels». À partir de corpus constitués d'articles issus de la presse économique et des rapports annuels de ces deux firmes, nous entendons délimiter les contours de la notion de média audiovisuel à la lumière des mutations induites par la montée en puissance des offres des plateformes SVOD.
\end{abstract}

Mots-clés : plateformes SVOD, médias audiovisuels, stratégies financières et éditoriales, Disney, Netflix.

Abstract: Our contribution focuses on the financial and editorial strategies of Disney and Netflix in the audiovisual market, in order to better understand the changes underway. This analysis aims to understand the forms of reciprocal adjustment between the market of SVOD platforms and that of "traditional" audiovisual media. Using articles from the economic press and annual reports from these two firms, we propose a definition of the notion of audiovisual media that takes into account changes brought about by the increasing influence of SVOD platforms offers.

Keywords: SVOD platforms, audiovisual media industry, financial and editorial strategies, Disney, Netflix.

Resumen: El texto analiza las estrategias financieras y editoriales implementadas por Disney y por Netflix con el objetivo de entender la lógica de las mutaciones que atraviesan el mercado audiovisual. Este análisis busca comprender las formas de ajustes recíprocos entre el mercado de las plataformas SVOD y el de los medios audiovisuales 


\section{Lucien PERTICOZ}

"tradicionales". A partir de artículos publicados en la prensa económica y de los informes anuales de estas dos empresas, se estudian los cambios producidos por la creciente oferta de las plataformas SVOD y se propone una nueva definición de medios audiovisuales.

Palabras claves: plataformas SVOD, medios audiovisuales, estrategias financieras y editoriales, Disney, Netflix. 
Filière de l'audiovisuel et plateformes SVOD : une analyse croisée des stratégies de Disney et Netflix

Depuis le mitan des années 2000 et la montée en puissance progressive de différents types d'offre de visionnage de vidéos en streaming, la production, la diffusion et la consommation de contenus audiovisuels semblent connaître un nouvel essor, alors même que les positions acquises des acteurs historiques de la filière apparaissent fragilisées. Dès lors, dans un environnement socioéconomique où les stratégies mises en œuvre par les acteurs des industries culturelles et ceux issus du Web donnent le sentiment d'évoluer à grande vitesse, il s'avère pertinent de procéder à une réduction du champ de l'analyse, afin de bien identifier certaines logiques sociales des processus à l'œuvre. En d'autres termes, la multidimensionnalité de ces industries (Miège, 2017) nous invite, au préalable, à faire preuve d'une certaine modestie méthodologique au moment d'appréhender certaines des mutations qui les traversent.

De ce fait, proposer une étude croisée de l'évolution des stratégies des firmes Walt Disney Company et Netflix peut sembler en contradiction avec le postulat que nous venons d'énoncer. En effet, il s'agit là d'acteurs qui saturent l'actualité médiatique et leur positionnement à une échelle mondiale peut aisément amener le chercheur à formuler des propositions par trop générales. Cependant, nous soutenons que cette approche est heuristique pour au moins deux raisons.

Premièrement, en nous focalisant sur la notion de filière (celle de l'audiovisuel ${ }^{1}$ dans le cas de la présente contribution) pour analyser les mutations induites par la montée en puissance des plateformes culturelles numériques, nous entendons nous prémunir contre les approches et les discours, scientifiques ou non, qui envisagent celles-ci uniquement sous

\footnotetext{
${ }^{1}$ Pour des raisons à la fois socioéconomiques, culturelles et historiques, les filières du cinéma et de la télévision ne peuvent être confondues ou assimilées l'une à l'autre. Néanmoins, dans le cadre de la présente contribution, nous ne nous intéresserons aux films de cinéma qu'au moment de leur disponibilité sur les plateformes culturelles numériques. En d'autres termes, nous élargissons le champ de l'analyse à l'ensemble des fictions audiovisuelles, qu'il s'agisse de séries ou de films (Guibert, Rebillard et Rochelandet, 2016), pour ensuite mieux le réduire aux stratégies des seuls opérateurs des plateformes susmentionnées.
} 


\section{Lucien PERTICOZ}

l'angle de la rupture radicale et du changement de paradigme. Ainsi, lorsque Pénard et Rallet (2014), dans le cadre d'une perspective qui excède celle des seules industries culturelles, estiment que «nous assistons à une reconfiguration des industries, auparavant organisées selon des lignes sectorielles verticales, aujourd'hui recomposées par des écosystèmes aux configurations complexes » (p. 88), il apparaît que ces auteurs sous-estiment précisément, comme nous le verrons, la prégnance du fonctionnement desdites industries «selon des lignes sectorielles verticales ", c'est-à-dire dans le cadre d'une logique de filière. Par ailleurs, dans le cas de Disney, Wasko (2001) a également démontré que, dès ses origines, cette firme a su développer des "écosystèmes aux configurations complexes " autour des nombreuses licences qu'elle détient. Ce constat nous amène dès lors à nuancer tout discours qui postulerait une rupture radicale entre un avant et un après l'émergence des plateformes culturelles numériques, ce qui passe nécessairement par une analyse s'inscrivant dans une perspective qui ne peut être que celle du temps long.

II en va à cet égard de même, selon nous, de la supposée opposition entre old et new media ainsi que de la notion de convergence telles que Jenkins (2008) les envisage. Entraînant nombre de chercheurs dans son sillage au milieu des années $2000^{2}$, Jenkins pensait ainsi pouvoir anticiper une convergence des médias devant aboutir à un effacement des frontières entre les filières historiques des industries culturelles, cette fusion inéluctable devant donner naissance à une sorte de métafilière : le transmédia (Jenkins, 2008). Or nous postulons, à l'inverse, que c'est précisément à partir d'une analyse par filières, en prenant en compte les spécificités socioéconomiques de chacune d'entre elles, qu'il devient possible d'appréhender finement les relations qu'elles entretiennent entre elles, non en annonçant la disparition - pour l'instant bien hypothétique et surtout non vérifiée - de leurs traits spécifiques " historiques " (Bouquillion, Miège et Mœglin, 2013). De plus, bien que les plateformes occupent indéniablement une place grandissante au sein des différentes filières des industries culturelles, "la perspective d'un modèle trans-filière efficient de la distribution et de la diffusion des

${ }^{2}$ En France, Bourdaa (2013) fait partie des « passeurs » les plus actifs des travaux de Jenkins. 
Filière de l'audiovisuel et plateformes SVOD : une analyse croisée des stratégies de Disney et Netflix

contenus numériques semble encore prématurée » (Bullich et Guignard, 2014b, p. 210).

Deuxièmement, en circonscrivant le champ de notre étude aux firmes Disney et Netflix, nous faisons le choix de focaliser notre contribution uniquement sur des acteurs proposant ou prévoyant de proposer un service de vidéo à la demande par abonnement $\left(S_{V} \mathrm{OD}^{3}\right)$. Nous pensons éviter ainsi la tentation qui consisterait à vouloir embrasser la totalité de ce que peut recouvrir la notion de plateformes culturelles numériques. En effet, s'il est toujours question de contenus audiovisuels, les modèles socioéconomiques de YouTube et de Netflix se distinguent nettement l'un de l'autre. Le premier s'inscrit dans la stratégie globale de Google et relève du modèle dit du « courtage informationnel » (Guibert, Rebillard et Rochelandet, 2016 ; Mœglin, 2007 ; Smyrnaios, 2017), alors que le second peut être vu comme une hybridation des logiques de production des contenus propres au modèle éditorial et de celles du modèle du club pour ce qui concerne leur diffusion/commercialisation ${ }^{4}$ (Miège, 2017 ; Perticoz, 2012). Si nous devions analyser ces stratégies en termes de modèles d'affaires (Benghozi, 2012), le constat demeurerait à cet égard identique. En effet, qu'il s'agisse de la structure de leur offre (économie de la fonctionnalité versus économie axée sur la diffusion de contenus premium), des modèles de revenus (publicitaire versus souscription) ou du type de relation aux clients (annonceurs versus abonnés), il existe des différences majeures entre YouTube et Netflix, dans la manière dont ils entendent s'approprier la valeur créée par le visionnage de contenus audiovisuels.

Dans ce contexte, les analyses croisées des stratégies de Disney - en tant qu'acteur historique des industries culturelles «traditionnelles » - et Netflix - en tant que pure player doivent nous permettre de faire ressortir ce qui les différencie, mais également doit nous fournir un certain nombre de similitudes en mesure d'aboutir à une première ébauche de définition d'un média audiovisuel, à l'ère des plateformes

\footnotetext{
${ }^{3}$ Par commodité, nous utiliserons l'acronyme anglo-saxon (SVOD pour Subscription Video on Demand), celui-ci étant le plus couramment employé en France.

${ }^{4}$ Dans le cas de la SVOD, l'usager-consommateur peut effectivement, moyennant le paiement d'un abonnement forfaitaire, accéder à l'ensemble du catalogue de titres dont l'opérateur de la plateforme détient les droits.
} 


\section{Lucien PERTICOZ}

culturelles numériques.

Du point de vue de la méthodologie employée, notre approche s'inscrit dans la continuité des travaux en économie politique de la communication (Miège, 2004). Nous interrogeons notamment les stratégies de ces deux firmes en nous appuyant sur les notions de modèles génériques (éditorial, de flot ainsi que leurs déclinaisons), ce qui nous permettra, en retour, d'en éprouver la pertinence ou les limites (Miège, 2017 ; Mœglin, 2007 ; Perticoz, 2012). Pour ce faire, nous nous appuierons sur deux types de corpus.

Le premier est constitué des rapports d'activité annuels de Disney et Netflix depuis $2007^{5}$. Nous en avons extrait un certain nombre d'indicateurs financiers (chiffre d'affaires, bénéfices nets, endettement à long terme et montant des actifs détenus) dont nous avons ainsi pu suivre l'évolution. Nous les avons ensuite croisés pour dégager les grandes orientations stratégiques de ces deux acteurs. Notre deuxième type de corpus regroupe des articles du quotidien de presse économique Les Échos. Nous l'avons constitué par la base de données Factiva, sur une période allant du $1^{\mathrm{er}}$ janvier 2007 au 23 janvier 2019, en retenant uniquement les articles où apparaissaient à la fois les termes Disney et Netflix ${ }^{6}$. Notre objectif était ici de faire ressortir une possible convergence de leurs stratégies financières et éditoriales. Enfin, soucieux de disposer d'une vue plus précise de celles-ci sur la période récente, nous avons constitué deux autres corpus d'articles, sur un intervalle allant du $1^{\mathrm{er}}$ janvier $2014^{7}$ au 23 janvier 2019 : l'un uniquement avec le terme Disney ${ }^{8}$ et l'autre uniquement avec le terme Netflix ${ }^{9}$. Ces articles nous ont ainsi permis d'appréhender finement l'évolution de deux types de stratégies fondamentalement liées. Tout d'abord celle des stratégies éditoriales mises en œuvre ainsi que les modalités de constitution de catalogues de ces deux firmes (programmation sous licence, rachats de concurrents, production de contenus

${ }^{5}$ C'est-à-dire l'année durant laquelle Netflix a proposé, pour la première fois, sa plateforme de SVOD aux États-Unis.

${ }^{6}$ Soit 139 articles de presse au total, sur la période considérée.

${ }^{7}$ C'est-à-dire l'année durant laquelle Netflix a lancé son service en France.

${ }^{8}$ Soit 239 articles de presse au total, sur la période considérée, Factiva permettant de réduire les résultats aux articles portant uniquement sur la firme "The Walt Disney Company $"$.

${ }^{9}$ Soit 290 articles de presse au total, sur la période considérée. 
Filière de l'audiovisuel et plateformes SVOD : une analyse croisée des stratégies de Disney et Netflix

originaux, etc.); ensuite, celle des stratégies financières déployées par ces deux acteurs pour y parvenir (autofinancement, recours à la dette bancaire, recours aux marchés financiers, élargissement du capital, échanges d'actions, etc.), ce qui nous amènera notamment à interroger la part de risque que ces stratégies comportent.

Afin d'exposer nos premiers résultats et les pistes ouvertes par notre recherche, la suite de notre contribution se déroulera en deux temps. Tout d'abord, nous reviendrons rapidement sur les trajectoires respectives de Disney et Netflix. Nous insisterons ainsi plus particulièrement sur les points de convergence que nous avons dégagés entre leurs stratégies. Ceci nous amènera ensuite, dans une deuxième partie, à interroger les mutations récentes d'un capitalisme médiatique où l'émergence des plateformes de SVOD (et surtout de Netflix) viennent bousculer les positions acquises des acteurs historiques, sans, à notre sens, remettre fondamentalement en cause certaines des logiques sociales les plus prégnantes qui structurent la filière de l'audiovisuel depuis des décennies. En conclusion, nous ouvrirons notre contribution sur l'avenir possible des médias audiovisuels qui, envisagé dans le temps long, ne fait selon nous que prolonger/renforcer les logiques sociales en question.

\section{Disney et Netflix : des origines différentes pour un même point d'arrivée ?}

Dès lors qu'il s'agit d'évoquer conjointement les stratégies financières et éditoriales de Disney et Netflix, notre corpus presse fait ressortir toute une rhétorique relevant d'une opposition frontale entre ces deux acteurs. Ceux-ci sont présentés, d'un côté, comme l'un des plus éminents représentants de l'industrie hollywoodienne et, de l'autre, comme un des fleurons de la Silicon Valley et de la « nouvelle économie ». Cette narration économique emprunte de " destruction créatrice » schumpétérienne (Schumpeter, 2004) invite donc son lectorat - par des titres tels que "Disney-Fox : la riposte d'Hollywood face à la Silicon Valley » ${ }^{10}$, «Disney

\footnotetext{
${ }^{10}$ Madelaine, N. (2017, 15 décembre). Disney-Fox : la riposte d'Hollywood face à la Silicon Valley. Les Échos, p. 01.
} 


\section{Lucien PERTICOZ}

pourrait muscler son offre "anti Netflix" avec Fox »" ou "Disney déclare la guerre à Netflix ${ }^{12}$ - à envisager ces deux acteurs en tant qu'idéal-types de la filière audiovisuelle " historique », pour Disney, et de l'économie numérique, pour Netflix, c'est-à-dire du « vieux monde » des médias " traditionnels » qui tenteraient de résister aux diverses remises en question de leurs modalités de valorisation des contenus audiovisuels, jugées obsolètes, par les acteurs issus du Web. Cette manière de présenter les stratégies à l'œuvre sous l'angle de l'ancien (les studios) contre le nouveau (les pure players du Web) s'inscrit, à cet égard, dans une certaine continuité des discours promotionnels qui ont accompagné les acteurs du Web collaboratif tout au long des années 2000 (Bouquillion et Matthews, 2010).

Toutefois, il convient tout d'abord de rappeler que cette situation de "guerre de mouvements conduite par des acteurs pour s'accaparer des flux de valeur nouvellement créés et réorienter à leur profit les flux de valeur existants " (Weygand, 2008) est aux fondements de la dynamique même du capitalisme et ne représente pas, à proprement parler, une nouveauté. Dès lors, c'est peut-être bien davantage à l'aune de cette dynamique qu'il convient d'analyser les stratégies respectives de Disney et Netflix, plutôt qu'en tentant de mettre au jour un supposé nouveau paradigme au sein (ou en dehors) de la filière audiovisuelle. En d'autres termes, toute tentative de compréhension de ces stratégies qui méconnaîtrait les logiques de mise en valeur du capital qui les sous-tendent prend le risque de passer à côté d'un de leurs plus puissants facteurs explicatifs (George, 2014 ; Mosco, 1998).

Ensuite, toute l'histoire des industries culturelles et des médias, depuis leurs origines, doit être envisagée dans le cadre d'une logique qui est avant tout celle de la " coopétition » entre acteurs et nouveaux acteurs du secteur (Bouquillion, 2012; Bullich et Guignard, 2014a), c'est-à-dire de stratégies qui alternent entre oppositions et alliances, où "c'est toujours contre l'acteur fort du moment que s'opèrent ces [dernières] » (Bouquillion, 2012, paragr. 27). Ceci doit notamment nous

\footnotetext{
${ }^{11}$ Alcaraz, M. (2017, 8 novembre). Disney pourrait muscler son offre " anti Netflix » avec Fox. Les Échos, p. 25.

12 Madelaine, N. (2017, 10 août). Disney déclare la guerre à Netflix. Les Échos, p. 17.
} 
Filière de l'audiovisuel et plateformes SVOD : une analyse croisée des stratégies de Disney et Netflix

amener à éviter toute essentialisation des stratégies des acteurs selon leur secteur d'activité d'origine et à plutôt porter notre réflexion au niveau de l'évolution dans le temps des rapports de force entre eux, mais également de celle de leurs intérêts respectifs.

Ainsi, Disney n'a pas systématiquement été dans une logique de confrontation directe vis-à-vis de Netflix. En effet, la plateforme SVOD de celle-ci a tout d'abord pu être considérée comme une source supplémentaire de revenus et un moyen de peser face à Google/YouTube en y diffusant des contenus exclusifs issus des licences détenues par Disney. C'était d'ailleurs tout l'enjeu de l'accord signé en décembre $2012^{13}$, accord qui permettait à Netflix de diffuser en exclusivité certaines fictions audiovisuelles de Disney et qui n'a finalement pas été renouvelé en 2017. En conséquence, Disney est en train de retirer, depuis fin 2018, l'essentiel de ses contenus de l'offre de Netflix - devenue entre-temps «l'acteur fort du moment " - en prévoyant de lancer sa propre plateforme SVOD (Disney+) d'ici la fin de l'année 2019 ${ }^{14}$. En effet, lorsqu'en 2011, Netflix a entamé son tournant stratégique en acquérant les droits de diffusion exclusifs de la série House of Cards ${ }^{15}$, elle ne représentait pas encore une menace pour les acteurs traditionnels de la filière audiovisuelle. Ce n'est que depuis 2015, au moment où la décision a été prise d'investir massivement dans la production de contenus exclusifs ${ }^{16}$, que Netflix s'est positionnée explicitement comme un concurrent direct de ceux-ci, non plus seulement en ce qui concerne la diffusion/distribution, mais également la détention d'un catalogue de titres premium dans le domaine des fictions audiovisuelles (qu'il s'agisse de séries télévisées ou, plus récemment, de films ${ }^{17}$ ).

Ce rapide retour sur l'évolution des choix stratégiques respectifs de Disney et Netflix au cours des dernières années

\footnotetext{
${ }^{13}$ De Meyer, K. (2012, 6 décembre). Avec Disney, Netflix bouscule la télé payante. Les Échos, p. 22.

${ }^{14}$ Madelaine, N. (2018, 20 novembre). Les chaînes françaises menacées dans leur accès aux productions américaines. Les Échos, p. 22.

15 Amiot, M. (2011, 21 mars). Netflix propose en exclusivité « House of Cards ». Les Échos, p. 30.

${ }^{16}$ Conesa, E. (2015, 15 septembre). Six milliards investis dans les contenus en 2016. Les Échos, p. 24.

${ }^{17}$ Madelaine, N. (2018, 22 mai). Netflix absent de la compétition à Cannes mais pas du marché. Les Échos, p. 25.
} 
appelle dès lors quelques commentaires sur le "devenir » de chacune de ces deux firmes. En effet, au-delà des confrontations que nous venons d'énoncer succinctement, il nous semble surtout que ces différents événements marquent une forme de convergence stratégique entre ces deux acteurs. Plus précisément et depuis 2015, il apparaît que Disney et Netflix tentent, chacun de leur côté, de combler ce qu'ils ont identifié comme leurs manques respectifs pour pouvoir se positionner comme le "média audiovisuel payant du $X X I^{e}$ siècle ", venant ainsi concurrencer directement les offres de télévision payante type "HBO " ou les bouquets de chaînes.

Avant de revenir plus en détail sur notre hypothèse, nous nous permettons de reprendre ici une longue citation de Bernard Miège (2007b), où l'auteur propose une définition de la notion de médias. Celui-ci évoque ainsi :

[des] dispositifs sociotechniques et sociosymboliques, basés de plus en plus sur un ensemble de techniques (et non plus sur une seule technique comme primitivement) permettant d'émettre et de recevoir des programmes d'information, de culture et de divertissement, avec régularité sinon de plus en plus en permanence, dans le cadre d'une économie de fonctionnement qui leur est propre [...], et dont la mise en œuvre est assurée par des organisations aux spécificités marquées [...], à destination de publics dont les caractéristiques sont plus ou moins stabilisées (p. 106).

En repartant de cette proposition, il ressort qu'au début des années 2010, au moment où une offre de plateformes SVOD commençait à se constituer, Disney pouvait schématiquement être envisagée en tant que producteur/fournisseur de " programmes d'information, de culture et de divertissement ", alors que Netflix, pour sa part, proposait « un ensemble de techniques [...] permettant d'émettre et de recevoir " ceux-ci, les algorithmes de recommandation développés par cette firme étant au centre de sa stratégie pour devenir un acteur majeur de la prescription de contenus audiovisuels. En ce sens, leurs positionnements respectifs semblaient se conformer aux modélisations d'auteurs qui considèrent que l'une des caractéristiques fondamentales de l'économie des plateformes se situe sur le plan d'une reconfiguration de l'intermédiation, 
Filière de l'audiovisuel et plateformes SVOD : une analyse croisée des stratégies de Disney et Netflix

processus qui aboutirait à un "découplage entre le marché reliant l'offreur et le prescripteur [...] et celui reliant le prescripteur au consommateur " (Benghozi et Paris, 2014, p. 78). Benghozi et Paris considèrent ainsi, dès le début des années 2000, que "l'intermédiation implique la prescription » (Benghozi et Paris, 2003, p. 225) et que c'est autour de celle-ci que se structurerait l'offre de contenus audiovisuels. Ces auteurs distinguent dès lors trois types de modèles d'affaires, dont deux sont susceptibles de nous intéresser dans le cadre de notre propos: les modèles d'affaires qu'ils nomment de " distribution » et " éditorial » (Lyubareva, Benghozi et Fidele, $2014)^{18}$. Dans le cas de la « distribution », la plateforme finance des contenus payants qu'elle diffuse en exclusivité, alors que le modèle dit « éditorial ${ }^{19}$ consiste davantage en un travail de sélection/diffusion/mise en visibilité (ou d'« éditorialisation ») de contenus professionnels spécifiques (fictions audiovisuelles, documentaires, jeux vidéo, etc.).

Or, dans le cas de la présente analyse, cette distinction entre ces deux modèles mérite d'être discutée, car, aujourd'hui, où placer Netflix si ce n'est à l'intersection entre modèle de " distribution » et modèle " éditorial »? En somme, si, au lancement de sa plateforme SVOD en 2007, Netflix pouvait être catégorisée du côté du modèle d'affaires "éditorial " confirmant ainsi l'hypothèse d'un découplage des marchés énoncée par Benghozi et Paris (2014) -, les investissements massifs dans les contenus audiovisuels auxquels cette firme a consenti depuis 2016 nous conduisent maintenant à considérer plutôt Netflix comme un média, au sens où l'entend Miège. En d'autres termes, il ne s'agirait pas tant de s'interroger sur la disparition prochaine des médias audiovisuels au profit des plateformes SVOD, mais bien davantage de comprendre comment celles-ci participent d'une reconfiguration des premiers, sans pour autant remettre fondamentalement en question la manière dont il convient de les définir.

L'analyse des stratégies mises en œuvre par Disney nous semble, à cet égard, venir confirmer notre propos. Cette firme

\footnotetext{
18 Les auteurs distinguent également la classe de modèles d'affaires dite " participative », qui recouvrent les plateformes agrégeant et diffusant «gratuitement » des contenus amateurs tel YouTube.

${ }_{19}$ Qu'il convient de bien distinguer du modèle générique (ou socioéconomique) éditorial (Miège, 2017 ; Mœglin, 2007 ; Perticoz, 2012).
} 


\section{Lucien PERTICOZ}

possèdait déjà de nombreux actifs dans les médias audiovisuels (ABC Television Group, ESPN, ainsi que $33 \%$ de la plateforme de SVOD Hulu). Le rachat d'une partie des actifs de la $21^{\text {st }}$ Century Fox doit notamment lui permettre de renforcer son catalogue de droits pour être en mesure, dès 2019, de lancer une offre SVOD à même de concurrencer Netflix. À cet égard, il n'est pas neutre que, parmi les actifs que Disney récupère à l'issue de cette transaction, se trouvent notamment les $33 \%$ de la joint-venture Hulu détenus par $21^{\text {st }}$ Century Fox, ce qui en fait l'actionnaire majoritaire de cette plateforme de SVOD, avec $66 \%$ des parts.

Ce rachat s'inscrit donc dans la continuité des mouvements récents de Disney qui, dès 2015, envisageait déjà de proposer une offre concurrente à celle de Netflix ${ }^{20}$. En cela, Disney n'entend donc pas se cantonner au seul rôle de fournisseurs de contenus premium pour les plateformes (SVOD ou de type "YouTube ») déjà existantes, mais bel et bien de continuer à s'affirmer en tant que média audiovisuel à part entière, ce qui passe notamment par une maîtrise de l'aval de la filière. En d'autres termes, alors même que les techniques d'émissionréception (et les usages et les pratiques de consommateurs auxquels elles donnent lieu) sont en pleine mutation, Disney continue de dessiner une stratégie qui maintient un couplage fort entre offre, prescription et consommation de programmes audiovisuels.

Au final, il apparaît que Disney n'a pas renoncé à être ce que Netflix est peut-être en passe de devenir : un média audiovisuel à vocation mondiale, à l'ère des plateformes culturelles numériques.

\section{Capitalisme médiatique, contenus audiovisuels et plateformes: quelles ruptures pour quelles continuités?}

À ce stade de notre proposition, nous nous appuierons pour partie sur un certain nombre de données financières extraites des rapports annuels de Disney et Netflix. L'objectif est ici de les confronter aux hypothèses précédemment énoncées et de

${ }^{20}$ Madelaine, N. (2015, 22 octobre). Disney va concurrencer Netflix en distribuant directement ses contenus. Les Échos, p. 24. 
Filière de l'audiovisuel et plateformes SVOD : une analyse croisée des stratégies de Disney et Netflix

voir dans quelle mesure elles peuvent ou non les vérifier. L'objectif de cette partie est également d'interroger la pertinence des modèles génériques (Miège, 2017 ; Perticoz, 2012) dans leur capacité à appréhender les mutations à l'œuvre au sein des médias et des "nouveaux" médias audiovisuels, mais également de contribuer à l'identification des logiques qui les sous-tendent.

En France, Netflix a annoncé, fin septembre 2018, que le nombre de ses abonnés en France s'élevait à 3,5 millions ${ }^{21}$. Néanmoins, bien qu'en nette progression, la part de la SVOD dans les dépenses en programmes audiovisuels des foyers français n'était, en 2016, que de 1,7\% (contre 0,4\% en 2014 et $1 \%$ en 2015) alors même que la télévision représentait encore $70,3 \%$ desdites dépenses ${ }^{22}$. De fait, ces données chiffrées illustrent toute la difficulté à laquelle le chercheur est confronté au moment de les mettre en perspective. Alors même que les positions des acteurs évoluent rapidement, le fait que les données collectées s'inscrivent dans des temporalités différentes ne peut que contribuer à complexifier l'exercice.

Dès lors, notre questionnement, dans le cadre de cette partie, sera double. Tout d'abord, est-il encore pertinent de raisonner en termes de médias au moment où les plateformes SVOD prennent une place grandissante dans les modes de consommations audiovisuelles? Ensuite, pouvons-nous considérer que ces médias ou "nouveaux" médias fonctionnent selon des logiques radicalement différentes de celles identifiées jusqu'à présent? II va de soi que les premières conclusions énoncées ci-dessous demanderont à être complétées, à mesure que les stratégies des acteurs étudiés produiront leurs effets. II n'en demeure pas moins que nos premiers résultats font déjà ressortir certaines permanences au sein de ce qu'il convient encore d'appeler le « capitalisme médiatique ».

\footnotetext{
${ }^{21}$ Madelaine, N. (2018, 28 septembre). Netflix tisse sa toile en France. Les Échos, p. 17.

${ }^{2}$ CNC (2018, 12 mai). La vidéo à la demande par abonnement en France : marché et stratégies des acteurs.
} 


\section{Lucien PERTICOZ}

\subsection{Logique de catalogue versus logique de recommandation}

En première analyse, les firmes Disney et Netflix ont ceci de commun qu'elles ont très clairement mis au centre de leur stratégie la détention d'un large catalogue de contenus audiovisuels premium. Pour atteindre cet objectif, Disney et Netflix ont consenti à des investissements massifs, que ce soit dans le cadre de rachats de licences ou dans la conception de contenus originaux. Pour 2018, Netflix a ainsi porté à 12 milliards de dollars US ses investissements dans la production/achat de programmes originaux ${ }^{23}$ (pour des investissements compris entre 5 milliards et 6 milliards en 2016 puis en 2017). La Figure 1 illustre ainsi fort bien cette tendance puisqu'elle fait apparaître que, sur le montant total de ses actifs, qui s'élevait à un peu plus de 19 milliards de dollars US en 2017, la part des contenus représentait à elle seule près de 14,7 milliards, soit plus de $77 \%$ du total. En cela, Netflix se différencie donc très nettement de plateformes telles que YouTube ou même Amazon Prime, pour qui la propriété de contenus exclusifs ne représente qu'une partie très minoritaire du total de leurs actifs. Netflix se positionne ainsi très clairement en tant que média et non plus seulement comme une simple place de marché permettant la valorisation marchande des programmes audiovisuels (et des publics qui les visionnent). Enfin, nous pouvons également constater que, dans une mesure significative, ces investissements ont été financés par la dette à long terme, celle-ci atteignant presque 6,5 milliards de dollars US en 2017. Comme nous le verrons dans la sous-partie suivante, cette situation comptable n'est pas sans entraîner quelques interrogations quant à l'avenir de Netflix à moyen terme.

\footnotetext{
${ }^{23}$ Madelaine, N. et Conesa, E. (2019, 21 janvier). Un record d'abonnés pour Netflix. Les Échos, p. 26.
} 
Filière de l'audiovisuel et plateformes SVOD : une analyse croisée des stratégies de Disney et Netflix

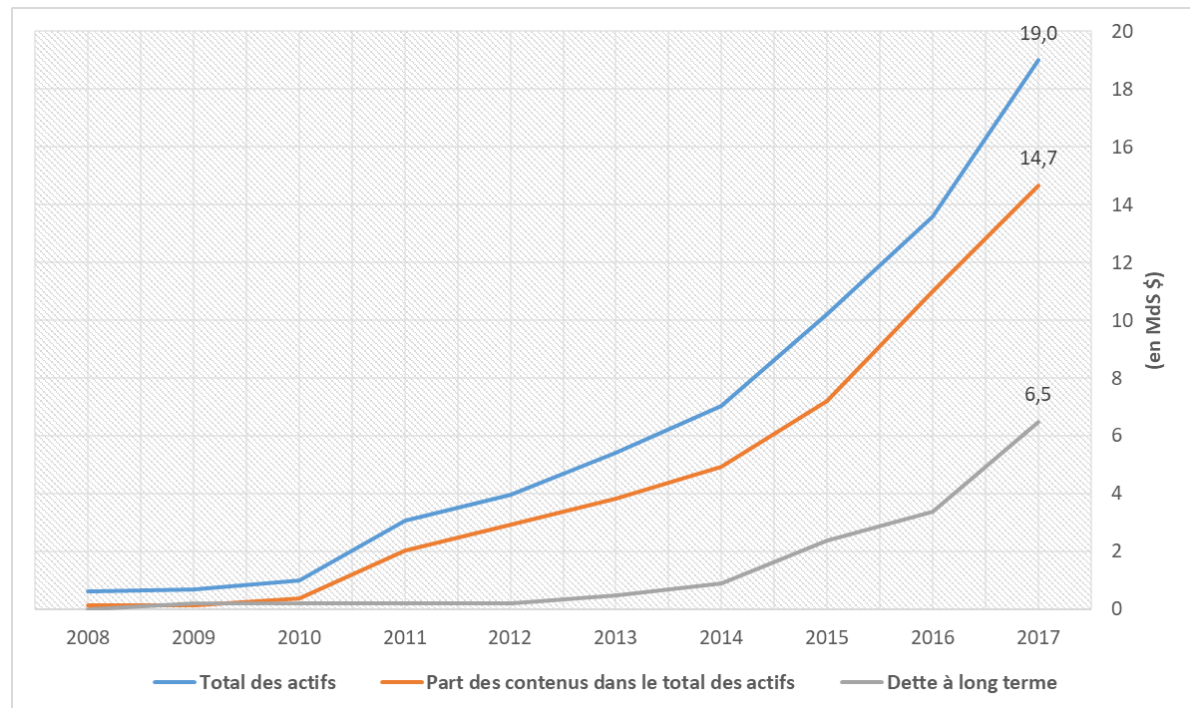

Figure 1. Évolution de la dette à long terme et du montant des actifs de Netflix ainsi que la part des contenus dans celui-ci, entre 2008 et 2017

De son côté et grâce à une offre de 71,3 milliards de dollars $\mathrm{US}^{24}$, Disney est parvenue à finaliser l'acquisition d'une grande partie des actifs détenus par la $21^{\text {st }}$ Century Fox, au premier rang desquels figurent les droits de ses catalogues télévisuel et cinématographique (composé de licences telles que X-Men, Avatar ou Les Simpson) ainsi que les $33 \%$ qu'elle détient dans le service de streaming Hulu ${ }^{25}$. Pour ce faire, et tout comme dans le cas de Netflix, Disney est dans l'obligation de recourir à la dette, son flux de trésorerie libre (free cashflow), qui s'élevait à 8,72 milliards de dollars US en $2017^{26}$, étant insuffisant pour financer l'intégralité de l'opération. Celleci doit ainsi lui permettre d'enrichir significativement son catalogue, tout en facilitant son entrée sur le marché des plateformes SVOD, et donc des outils de recommandation.

Avant de mettre en question la place occupée par ces outils dans les stratégies des acteurs considérés, il nous paraît utile

\footnotetext{
${ }^{24} \mathrm{Au}$ total, 85,1 milliards de dollars US, en incluant la reprise de la dette. Face à la concurrence de Comcast dans cette opération, Disney s'est ainsi vue obligée de réévaluer sa première offre, qui s'élevait déjà à 52,4 milliards de dollars US.

${ }^{25}$ Rauline, N. (2018, 21 juin). Face à Comcast, Disney améliore son offre sur Fox. Les Échos, p. 15.

26 Market Watch (2018). Annual Financials for Walt Disney Co. Repéré à https://www.marketwatch.com/investing/stock/dis/financials/cash-flow
} 


\section{Lucien PERTICOZ}

de revenir sur le rôle que les contenus sont amenés à jouer dans ce contexte. Ainsi, Miège indiquait dès la fin des années 1990, au moment où les débats tournaient autour d'une supposée convergence à venir entre les industries culturelles et le secteur des télécommunications :

deux éléments paraissent devoir être mis en avant: d'abord le souci de ne pas prendre de risques inconsidérés alors que l'on sait que la "construction » d'un média est une œuvre de longue haleine, faite d'essais/erreurs, et supposant toute une série de négociations avec les usagers et d'ajustements à leurs pratiques; ensuite la conscience, plus ou moins diffuse, que la question des programmes est essentielle à la progression de l'ensemble du secteur et que, tôt ou tard, des réponses devront lui être apportées, plus ou moins en conformité avec les logiques sociales qui sont à la source $\mathrm{du}$ fonctionnement des industries culturelles et informationnelles (de la conception des produits jusqu'à leurs usages) et qui se sont mises en place tout au long du $X X^{e}$ siècle (Miège, 2007a, p. 48).

Sur le premier élément soulevé par Miège, il apparaît au contraire que Disney et Netflix sont effectivement dans une phase de prise de risques financiers donnant lieu à des montages mêlant recours aux fonds propres de la firme, collecte de fonds auprès de sociétés de capital-risque, paiement en actions, etc. À ce stade, alors même que les analystes financiers oscillent entre inquiétude ${ }^{27}$ et optimisme ${ }^{28}$, il est bien évidemment encore un peu tôt pour savoir si ces prises de risques s'avèreront ou non « inconsidérés ». En revanche, ces stratégies s'inscrivent sans conteste dans le temps long des mutations de la filière audiovisuelle et, plus précisément, de celles de la télévision payante, dont elles reprennent le système par abonnement forfaitaire. En ce sens, les enjeux relatifs aux nouveaux outils de recommandation personnalisée ne se distinguent pas de ceux qui poussent les

\footnotetext{
${ }^{27}$ Moutot, A. (2018, 17 juillet). Netflix chute en Bourse après une progression du nombre d'abonnés moins forte que prévu. Les Échos.fr. Repéré à https://www.lesechos.fr/17/07/2018/lesechos.fr/0301988508331 netflix-chute-enbourse-apres-une-progression-du-nombre-d-abonnes-moins-forte-que-prevu.htm

${ }^{28}$ Mediavilla, L. (2019, 14 janvier). Netflix : Morgan Stanley chiffre les futurs énormes bénéfices. Les Échos.fr. Repéré à https://www.lesechos.fr/tech-medias/medias/0600 511885430-netflix-morgan-stanley-chiffre-les-futurs-enormes-benefices-2236186.php
} 
Filière de l'audiovisuel et plateformes SVOD : une analyse croisée des stratégies de Disney et Netflix

médias à tenter de cerner au mieux les habitudes de consommation de leurs publics. En d'autres termes, ces outils de recommandation jouent sensiblement le même rôle que celui de la grille de programmation pour le modèle de flot, à savoir celui d'outil d'encadrement des pratiques des publics (Miège, 2017 ; Perticoz, 2012), bien qu'il soit indéniable que l'élaboration et la mise à jour de ces outils aient passé un cap significatif en matière d'industrialisation, l'analyse supposément en "temps réel » des pratiques de visionnage des abonnés venant se substituer aux classiques analyses d'audience. Néanmoins, si nombre de discours d'acteurs (notamment politiques et culturels) font part d'une certaine inquiétude quant aux capacités prédictives des algorithmes qui régissent lesdits outils, des auteurs ont pu montrer que les interventions humaines étaient encore nombreuses pour assurer leur bon fonctionnement. Farchy, Méadel et Anciaux (2017) soulignent ainsi que «la puissance de la recommandation personnalisée repose [...] essentiellement sur les comportements de l'ensemble des utilisateurs scrutés en permanence par des opérateurs " et que celle-ci « n'est pas liée à la seule force technique de son algorithme » (p. 181).

En toute rigueur, nous assistons effectivement à une forme de délégation technique de la recommandation qui soulève d'ailleurs de légitimes questions, notamment sur le plan du respect de la vie privée et de la concurrence entre les différents contenus diffusés sur Netflix. Ainsi, nous pourrions nous demander si cette firme n'a pas tendance à favoriser la mise en avant des titres dont elle détient l'exclusivité. Toutefois, cet outil est loin de pouvoir se passer de toute intervention humaine, notamment lors du paramétrage des critères qui font «tourner » l'algorithme. Tout comme pour la grille de programmation, les outils de recommandation, s'ils doivent s'imposer, devront donc aussi passer par une «phase d'essais/erreurs " et de "négociation " avec les usagersconsommateurs. Ces considérations nous conduisent ainsi directement au deuxième élément avancé par Miège, concernant le caractère essentiel de «la question des programmes », question que les investissements massifs consentis par Disney et Netflix contribuent à remettre au centre des débats. 


\section{Lucien PERTICOZ}

En effet, si les enjeux relatifs aux nouveaux outils de recommandation ne peuvent être ignorés, force est d'admettre que c'est bien le catalogue proposé qui permet d'attirer de nouveaux utilisateurs et de collecter des données de qualité sur les comportements de ces derniers. Autrement dit, les contenus constituent toujours la "mise de départ" des médias audiovisuels dans leur stratégie de conquête des publics, ce qui nous ramène à la notion de risque, qui est consubstantielle aux industries culturelles, toutes filières confondues (Bouquillion, 2008 ; Miège, 2017 ; Mœglin, 2007). D'une certaine manière, en prenant pleinement sa part du risque structurel caractéristique des industries culturelles et en ne se limitant pas à la seule dimension technique de la recommandation, Netflix affirme ainsi une volonté stratégique de devenir le modèle de référence des médias audiovisuels, dans les prochaines années. C'est d'ailleurs bel et bien dans cette "guerre de positions ", dont les outils de recommandation ne constituent qu'une des armes, que Disney tente de maintenir sa capacité à capter la valeur générée par les licences qu'elle détient et les contenus qu'elle produit.

En définitive, qu'il s'agisse de Disney ou de Netflix, les différents points que nous venons de soulever nous amènent à soutenir que ces acteurs sont finalement bien loin d'une logique de "découplage » entre offre, prescription et consommation de contenus audiovisuels. Ces firmes entendent au contraire maîtriser l'ensemble de la chaîne, c'est-à-dire s'inscrire pleinement dans une logique industrielle de fonctionnement en filière, en mettant en œuvre une stratégie classique d'intégration verticale allant de l'amont à l'aval. En nous appuyant sur les modèles génériques pour analyser leur situation respective, nous pouvons considérer que Disney et Netflix déclinent le modèle éditorial vers une logique qui serait celle du club payant, ce qui implique de disposer d'un catalogue de contenus premium suffisamment attractif. À terme, si cette tendance se confirme, c'est bien les offres de télévision à péage de type "Canal+ " ou " $\mathrm{HBO}$ » qui risquent d'être les plus touchées par ces évolutions ${ }^{29}$. C'est en tout cas avec elles que les offres de Netflix (et bientôt de Disney) entrent le plus

\footnotetext{
${ }^{29}$ Alors même que Netflix semble s'inscrire dans une stratégie éditoriale comparable à celle dite de la "télévision de qualité " (Quality TV), cette dernière ayant, pour partie, contribué au succès commercial de l'offre de télévision payante de HBO, dans les années 1990 (McCabe et Akass, 2008).
} 
Filière de l'audiovisuel et plateformes SVOD : une analyse croisée des stratégies de Disney et Netflix

directement en concurrence. II ne s'agit pas là uniquement d'une concurrence entre acteurs (qui relève, somme toute, du " quotidien » du capitalisme médiatique), mais davantage d'une concurrence entre modèles économiques et types de médias audiovisuels (les plateformes SVOD face à la télévision payante), dont les modes de financement (le recours à l'abonnement) sont trop proches pour ne pas amener les consommateurs à devoir procéder à des arbitrages. À cet égard, il apparaît que, pour parvenir à s'imposer, la richesse des catalogues de même que la facilité des modalités techniques permettant d'y accéder seront des critères tout aussi déterminants (voire plus) que celui de la qualité de la recommandation personnalisée qui sera proposée par ces plateformes culturelles numériques. En ce sens, la question du mode de financement des contenus revêt dès lors un caractère tout à fait central.

\subsection{Internationalisation, concentration et financiarisation : des tendances renforcées par les plateformes}

Disney et Netflix se sont ainsi engagées dans ce qui s'apparente à une course pour renforcer leurs catalogues respectifs, ce qui passe par des investissements spectaculaires qui ne sont pas sans rappeler les mésaventures de Vivendi Universal et AOL Time Warner, à la fin des années 1990 (Bouquillion, 2008, 2012). Comme nous l'avons vu, l'arrivée de Netflix représente l'un des derniers avatars de «l'entrée [massive] des acteurs des industries de la communication dans l'économie des contenus " (Bouquillion, 2012, paragr. 33). Toutefois, à la différence de Google, Apple ou Facebook, Netflix ne se concentre pas uniquement sur l'aval de la filière audiovisuelle et tente, tout comme Disney, de profiter des leviers offerts par les marchés financiers pour parvenir à ses fins (notamment par le recours à des collectes de fonds auprès de sociétés de capital-risque). La financiarisation ne semble donc plus devoir être uniquement envisagée comme «un facteur clef de la dynamique des [seules] industries de la communication, dans leurs rapports internes comme avec d'autres acteurs économiques " (Bouquillion, 2012, paragr. 34.). En effet, les récents mouvements, tels que l'acquisition de la $21^{\text {st }}$ Century Fox par Disney (la moitié de 


\section{Lucien PERTICOZ}

l'opération étant financée en actions de la firme ${ }^{30}$ ) ou la dernière collecte de fonds de 2 milliards de dollars US opérées par Netflix à l'automne $2018^{31}$, illustrent ainsi le fait que des opérations financières de grande envergure, au sein des industries culturelles, sont déjà à l'œuvre.

Par ailleurs, ces investissements semblent s'inscrire dans une logique industrielle qui est bel et bien celle du fonctionnement des médias audiovisuels, c'est-à-dire celui de la filière. Ils se distinguent donc en cela des opérations menées dans le cadre du paradigme qui prévalait lors de la première "bulle Internet», celui d'une supposée convergence entre contenus médiatiques et réseaux de télécommunication. En d'autres termes, les synergies industrielles que tentent de faire émerger ces deux firmes diffèrent sensiblement de celles qui avaient la faveur des marchés à la fin des années 1990. Qu'il s'agisse de Disney ou de Netflix, ces deux acteurs visent une intégration verticale de l'amont à l'aval de la filière, afin d'obtenir ou de conserver une place dominante (ou des pouvoirs de marché) au sein du secteur des médias audiovisuels. Du point de vue des modèles génériques (Miège, 2017), Disney et Netflix ne s'écartent finalement pas de la logique du modèle éditorial. Dans le même temps, sur ce le plan socioéconomique (Guibert, Rebillard et Rochelandet, 2016; Mœglin, 2007), il ressort que la logique qui a leurs faveurs, à terme, pour financer la production/acquisition de programmes est bien celle de l'abonnement ${ }^{32}$.

II n'en demeure pas moins que, dans un premier temps, les moyens mis en œuvre pour y parvenir (le recours à la dette ou à l'achat en actions selon les cas) doivent être mis en regard des fondamentaux financiers de chacune de ces deux firmes. Du côté de Disney, la Figure 2 fait apparaître un chiffre d'affaires en constante progression depuis 2009 (avec un léger recul, en 2017, à 55,1 milliards de dollars US) et, surtout, un

${ }^{30}$ Conesa, E. (2018, 20 juillet). Disney a la voie libre pour racheter la Fox. Les Échos, p. 21.

31 Les Échos (2018, 23 octobre). Netflix va lever 2 milliards de dollars supplémentaires. Les Échos.fr. Repéré à https://www.lesechos.fr/23/10/2018/lesecho s.fr/0600020513402 netflix-va-lever-2-milliards-de-dollars-supplementaires.htm

${ }^{32}$ Différant en cela du mode de financement publicitaire privilégié par les acteurs du modèle de flot, ce qui place ces derniers en concurrence plus ou moins directe avec les firmes dont le modèle socioéconomique s'est construit autour du courtage informationnel (Google, Facebook, voire Amazon). 
Filière de l'audiovisuel et plateformes SVOD : une analyse croisée des stratégies de Disney et Netflix

bénéfice net qui atteint 13,1 milliards de dollars US sur l'exercice 2018. De plus, le niveau d'endettement à long terme est contenu à 17,1 milliards de dollars US sur la même période. En toute rigueur, il s'agit donc d'une firme dont les indicateurs financiers semblent des plus solides. À cet égard, la Figure 3 fait également apparaître que les contenus audiovisuels contribuent significativement à la rentabilité globale de Walt Disney Company ${ }^{33}$. En effet, le bénéfice net cumulé des divisions médias et studios atteint 9,6 milliards de dollars US en 2018. Enfin, l'ensemble des actifs du groupe est évalué à plus de 98 milliards de dollars US (parcs d'attractions inclus) alors que sa capitalisation boursière fluctue autour de 160 milliards de dollars US. Néanmoins, la montée en puissance des plateformes SVOD et, plus particulièrement, les offensives de Netflix dans les médias audiovisuels l'amènent à devoir pousser un peu plus loin la logique de concentration dans ce secteur. Ainsi, loin de remettre en question les logiques du capitalisme financier, l'arrivée des plateformes de SVOD ne fait que contribuer à les renforcer.

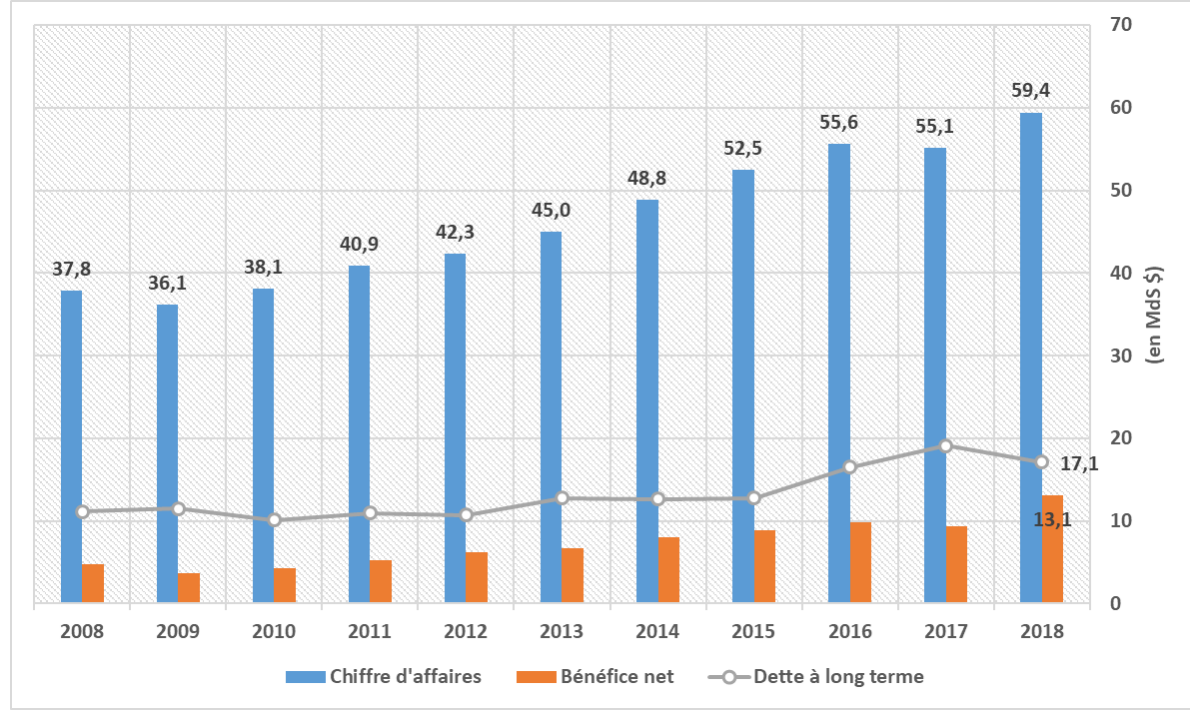

Figure 2. Évolution du chiffre d'affaires, du bénéfice net et de la dette à long terme de The Walt Disney Company, entre 2008 et 2018

\footnotetext{
${ }^{33}$ Voire compense les déficits de certaines de ses divisions, le bénéfice net cumulé des divisions médias et studios étant supérieur à celui de l'ensemble du groupe.
} 


\section{Lucien PERTICOZ}

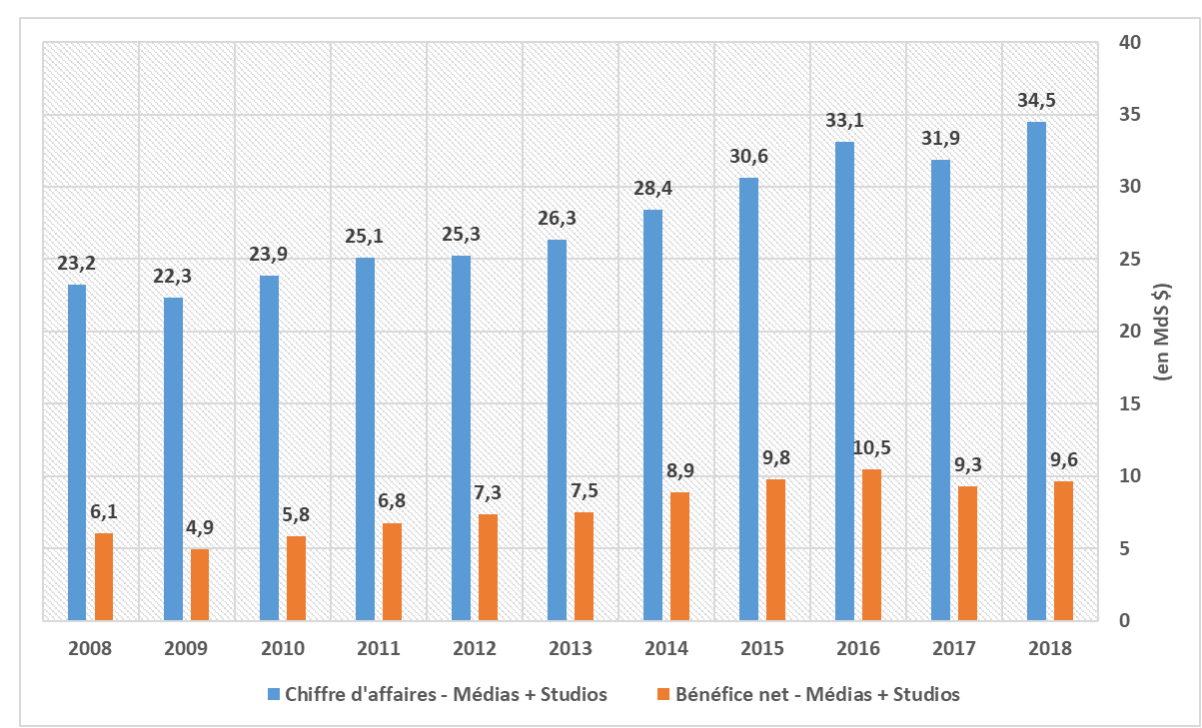

Figure 3. Évolution du chiffre d'affaires et du bénéfice net des divisions Media Networks + Studio Entertainment de The Walt Disney Company, entre 2008 et 2018

Ce constat est finalement assez proche de celui que nous pensons pouvoir formuler concernant les logiques de financiarisation. En effet, si le rachat de la $21^{\text {st }}$ Century Fox va effectivement dans le sens de davantage de concentration économique et peut s'entendre d'un strict point de vue industriel ${ }^{34}$, le montant de l'opération peut également amener à considérer cette opération comme un "coup financier » (Bouquillion, 2008).

Tout d'abord, la transaction est financée pour moitié par des actions de la firme Disney, alors même que certains fonds d'investissement tels que The Vanguard Group, BlackRock Fund Advisors ou SSgA Funds Management sont des actionnaires institutionnels importants de ces deux firmes. Cette acquisition leur permet donc de valoriser leurs participations au capital de la future entité, dans une opération où ces fonds sont à la fois vendeurs et acquéreurs. Ensuite, il apparaît également que ce type de transaction s'avère des plus lucratives pour les banques d'investissement qui conseillent ces firmes (telles que Goldman Sachs pour la $21^{\text {st }}$ Century Fox) et dont le montant

34 Des synergies éditoriales sont notamment envisagées en ce qui a trait aux licences Marvel dont une partie est détenue par la $21^{\text {st }}$ Century Fox. 
Filière de l'audiovisuel et plateformes SVOD : une analyse croisée des stratégies de Disney et Netflix

des commissions est indexé sur le montant final de l'opération $^{35}$. En tout état de cause, si la prise de risque est réelle pour Disney en tant que firme, il reste difficile, à ce stade, de savoir si ce sont les considérations financières ou industrielles qui l'emporteront dans le cadre de cette transaction.

La stratégie d'investissement massif de Netflix dans la production de programmes audiovisuels répond aux mêmes objectifs, à savoir dégager des pouvoirs de marché vis-à-vis de la concurrence (et ce, avant que l'offre SVOD de Disney ne soit opérationnelle). Toutefois, la question des risques qui menacent la firme ainsi que celle du poids des logiques de financiarisation qui pèsent sur sa stratégie se posent peut-être un peu différemment. En effet, Netflix n'est un acteur de la production de contenus audiovisuels que depuis très récemment. Dès lors, la constitution d'un catalogue en mesure de concurrencer Disney s'effectue à marche forcée, en ayant massivement recours à l'endettement. Netflix se doit ainsi de poursuivre sa croissance en nombre d'abonnés (qui s'élevait à 139 millions dans le monde en janvier $2019^{36}$ ), afin de maintenir son leadership sur le marché de la SVOD, de s'assurer des pouvoirs de marché dans le secteur et ainsi de pouvoir continuer à... s'endetter auprès des investisseurs financiers. D'une certaine manière, la confiance de ces derniers semble indexée sur celle affichée par Reed Hastings, CEO et Chairman de Netflix (et membre du conseil d'administration de Facebook). Or, comme le montre la Figure 4, le bénéfice net de Netflix ne s'élève qu'à un peu plus de 558 millions de dollars US en 2017, pour une dette à long terme d'un montant de 6,5 milliards de dollars US. Par ailleurs, les actifs de Netflix s'élèvent à 19 milliards de dollars US, dont $77 \%$ correspondent aux contenus qu'elle détient. Autrement dit, si "la "construction" d'un média est une œuvre de longue haleine " (Miège, 2007a, p. 48), Netflix semble pour sa part décidé à aller très vite et donc à s'appuyer sur les acteurs financiers pour atteindre ses

35 Conesa, E. (2018, 27 juin). Le buzz des États-Unis : Goldman empoche 100 millions de dollars dans la bataille pour les actifs de Fox. Les Échos.fr. Repéré à https://www.lesechos.fr/27/06/2018/lesechos.fr/0301883808449 le-buzz-des-etatsunis---goldman-empoche-100-millions-de-dollars-dans-la-bataille-pour-les-actifs-defox.htm

${ }^{36}$ Madelaine, N. et Conesa, E. (2019, 21 janvier). Un record d'abonnés pour Netflix. Les Échos, p. 26. 


\section{Lucien PERTICOZ}

objectifs. Dès lors, quand bien même sa stratégie semble se conformer à une logique industrielle cohérente, elle demeure toutefois largement tributaire des logiques spéculatives induites par la financiarisation du secteur des industries culturelles et des médias.

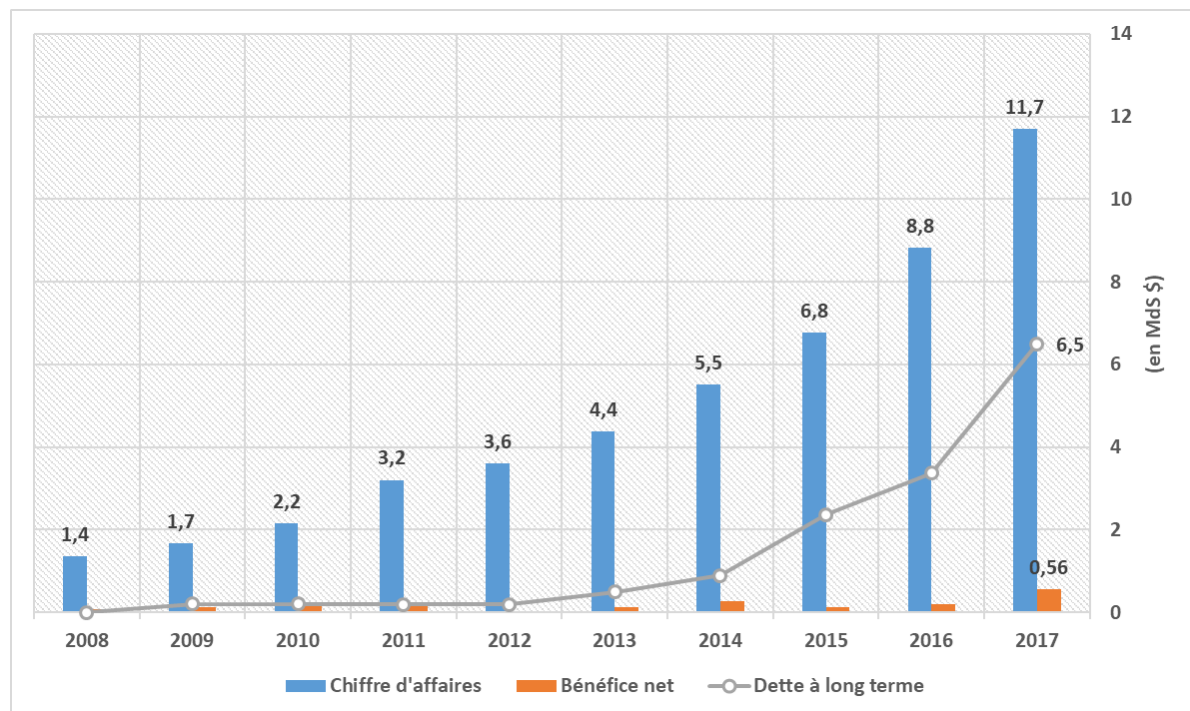

Figure 4. Évolution du chiffre d'affaires, du bénéfice net et de la dette à long terme de Netflix, entre 2008 et 2017

En mai 2017, la capitalisation boursière de Netflix a ainsi dépassé pour la première fois celle de Disney ${ }^{37}$, avant que la tendance ne s'inverse à la fin de l'année 2018. Compte tenu de la rentabilité respective des deux entités, ces fluctuations nous amène à nous demander dans quelle mesure ces valorisations n'ont pas un caractère essentiellement spéculatif. De plus, ces tendances sont de nature à renforcer encore un peu plus la dépendance de ces firmes vis-à-vis des marchés financiers, compte tenu du poids de leur " avis " au moment de négocier le financement de leurs investissements. En somme, Netflix est, d'une certaine manière, poussée au gigantisme et à la poursuite de sa croissance rapide à l'international ${ }^{38}$, si elle veut continuer à engranger des abonnés et ainsi bénéficier

37 Ibid.

${ }^{38}$ Madelaine, N. (2018, 20 avril). Netflix : la stratégie «tour de Babel ». Les Échos, p. 19. 
Filière de l'audiovisuel et plateformes SVOD : une analyse croisée des stratégies de Disney et Netflix

d'évaluations favorables des acteurs de la finance sur ces profits futurs ${ }^{39}$. Il en va de même pour Disney, qui doit également démontrer sa capacité à devenir un acteur dominant du marché de la SVOD. En tout état de cause, la rentabilisation éventuelle des investissements consentis ne peut être envisagée qu'à l'échelle mondiale, ce qui ne peut qu'entraîner la poursuite d'une logique, déjà bien engagée, d'internationalisation du capitalisme médiatique.

De plus, ce déploiement ne s'effectue pas de manière homogène et doit prendre en compte les spécificités culturelles et réglementaires locales, ce qui implique de diversifier son catalogue. Netflix, dès son arrivée en France, a ainsi fait part de sa volonté de produire des contenus en langue française, volonté que la firme a renouvelée en septembre 2018, en annonçant le lancement de sept nouvelles productions ${ }^{40}$. En revanche, il apparaît que la question de la chronologie des médias demeure un des principaux points bloquants ${ }^{41}$, Netflix préférant diffuser les films qu'elle produit directement sur sa plateforme SVOD, sans passer par une sortie en salles (du moins sur le territoire français). Alors même que Netflix s'affirme de plus en plus comme un acteur important de la filière cinématographique ${ }^{42}$, cette volonté de contrôler les fenêtres de diffusion des contenus qu'elle produit vient fragiliser tout le système de financement des films en France.

Quoiqu'il en soit, cette incertitude structurelle - déjà bien documentée - sur le plan des valeurs d'usage des programmes audiovisuels (Bouquillion, 2008 ; Miège, 2017 ; Mœglin, 2007) représente un facteur de coûts (et donc de risques) supplémentaire pour Disney et Netflix. En cela, la croissance des offres SVOD ne semble pas devoir échapper aux logiques, déjà anciennes, qui sous-tendent l'ensemble des filières des industries culturelles et des médias.

\footnotetext{
${ }^{39}$ Mediavilla, L. (2019, 14 janvier). Netflix : Morgan Stanley chiffre les futurs énormes bénéfices. Les Échos.fr. Repéré à https://www.lesechos.fr/tech-medias/medias/0600 511885430-netflix-morgan-stanley-chiffre-les-futurs-enormes-benefices-2236186.php ${ }^{40}$ Madelaine, N. (2018, 28 septembre). Netflix tisse sa toile en France. Les Échos, p. 17.

${ }^{41}$ Madelaine, N. (2018, 7 septembre). Accouchement difficile pour la chronologie des médias. Les Échos, p. 22.

${ }^{42}$ Alcaraz, M. (2019, 23 janvier). Netflix en lice pour les Oscars. Les Échos, p. 24.
} 


\section{Lucien PERTICOZ}

\section{Conclusion}

Finalement, comme nous l'avons souligné avec l'exemple de la firme Disney, les droits et les licences détenus par celle-ci ont d'emblée été envisagés dans une perspective que les économistes pourraient qualifier de " multiversants ». En effet, dès les années 1930, les personnages créés à l'époque ont rapidement été déclinés sous forme de produits dérivés, qu'il s'agisse de jouets ou, plus tard en 1955, du premier parc d'attractions de la firme (Wasko, 2001). En somme, c'est tout l'univers des franchises détenues par Disney ainsi que les droits de propriété intellectuelle qui s'y rattachent qui peuvent être considérés comme une plateforme, mais de nature avant tout sociale-symbolique. Dès lors, celle-ci peut effectivement être envisagée comme une place de marché immatérielle permettant de faciliter la "rencontre » de plusieurs types d'offres et de demandes, place de marché dont Disney est l'opérateur principal.

Dans cette optique et en toute rigueur, les services de SVOD ne relèvent pas de la rupture radicale avec l'« ancien monde " des industries culturelles et du capitalisme médiatique, mais constituent davantage une "nouvelle étape de l'industrialisation de la culture » (Matthews, 2015, p. 67). Car si, comme nous l'avons vu, Disney ambitionne d'être un acteur clé de la SVOD, Netflix, en retour, entend elle aussi faire fructifier le capital social-symbolique que constitue son catalogue, la commercialisation de produits dérivés étant l'une des pistes d'ores et déjà explorées ${ }^{43}$. En somme, en positionnant les contenus au centre de leurs stratégies, ce serait finalement les médias audiovisuels qui représenteraient l'avenir et l'horizon des plateformes culturelles numériques, et non l'inverse.

Si cette hypothèse se vérifie, il conviendra dès lors d'élargir le champ qui était celui de cette contribution. II y a élargissement, tout d'abord, aux autres acteurs qui entendent se positionner sur le secteur de la SVOD, qu'il s'agisse de câblo-opérateurs tels que AT\&T qui, après l'acquisition de Time Warner, aspire à faire de HBO un concurrent direct de Netflix et de Disney ${ }^{44}$, ou des acteurs "régionaux " tels que Canal+,

${ }^{43}$ Robequain, L. (2017, 9 février). Netflix va lancer des produits dérivés, à l'instar des grands studios de cinéma. Les Échos, p. 23.

${ }^{44}$ Che, J. (2018, 9 juillet). AT\&T veut que HBO ressemble davantage à Netflix. Les Échos, p. 21. 
Filière de l'audiovisuel et plateformes SVOD : une analyse croisée des stratégies de Disney et Netflix

qui souhaite relancer son offre SVOD ${ }^{45}$. L'un des objectifs serait notamment de se questionner sur la place des contenus dans la structure de leur offre au regard de la supposée montée en puissance d'une économie de la fonctionnalité (Benghozi, 2012).

Ainsi, il conviendra d'analyser comment ces offres s'insèrent dans les stratégies globales de chaque firme étudiée. À cet égard, si l'offre SVOD proposée par Amazon répond à des objectifs stratégiques s'inscrivant dans le cadre du modèle du courtage informationnel, il n'en demeure pas moins que, du point de vue du consommateur, Amazon Prime Video vient directement concurrencer Netflix et Disney+. II y a donc élargissement, ensuite, à l'ensemble des acteurs de la filière et notamment aux producteurs audiovisuels indépendants qui doivent faire face à une reconfiguration rapide des circuits de diffusion/distribution, ce qui n'est pas sans conséquence sur les modes de financement de la création. En d'autres termes, si la question des contenus demeure centrale, de quels types de contenus audiovisuels s'agit-il, comment sont-ils produits et par quels acteurs?

Et il y a élargissement, enfin, aux autres filières, le " modèle " Netflix semblant inspirer des acteurs tels que Microsoft qui ambitonne de devenir le « Netflix du jeu vidéo ${ }^{46}$ ". En définitive, dans un cadre où les logiques propres au capitalisme médiatique ne semblent pas être fondamentalement remises en question, les recherches sur les plateformes de streaming par souscription représentent un des points d'entrée permettant d'appréhender les rapports de force qui s'y jouent.

\section{Références}

Benghozi, P.-J. (2012). Entreprises culturelles et Internet: contenus numériques et modèles d'affaires innovants. Paris, France : ministère de la Culture et de la Communication.

\footnotetext{
45 Madelaine, N. (2019, 10 janvier). Canal+ repart à l'offensive face à Netflix. Les Échos, p. 1.

${ }^{46}$ Nedelec, G. (2019, 22 janvier). Microsoft affiche ses ambitions dans le jeu vidéo en streaming. Les Échos.fr. Repéré à https://www.lesechos.fr/techmedias/hightech/0600561603866-microsoft-affiche-ses-ambitions-dans-le-jeu-videoen-streaming-2238387.php
} 


\section{Lucien PERTICOZ}

Benghozi, P.-J. et Paris, T. (2003). De l'intermédiation à la prescription: le cas de l'audiovisuel. Revue française de gestion, (402), 205-227.

Benghozi, P.-J. et Paris, T. (2014). L'économie culturelle à l'heure du numérique: une révolution de l'intermédiation. Dans L. Jeanpierre et $\mathrm{O}$. Roueff (dir.), La culture et ses intermédiaires (p. 175-188). Paris, France: Éditions des Archives contemporaines.

Bouquillion, P. (2008). Les industries de la culture et de la communication. Les stratégies du capitalisme. Grenoble, France : Presses universitaires de Grenoble.

Bouquillion, P. (2012). Concentration, financiarisation et relations entre les industries de la culture et industries de la communication. Revue française des sciences de l'information et de la communication, (1). doi: 10.4000/rfsic.94

Bouquillion, P. et Matthews, J. (2010). Le Web collaboratif. Mutations des industries de la culture et de la communication. Grenoble, France : Presses universitaires de Grenoble.

Bouquillion, P., Miège, B. et Mœglin, P. (2013). L'industrialisation des biens symboliques. Les industries créatives en regard des industries culturelles. Grenoble, France : Presses universitaires de Grenoble.

Bourdaa, M. (2013). Le transmedia stroytelling. Terminal, (112), 7-10.

Bullich, V. et Guignard, T. (2014a). Les dispositifs de "TV connectée ». Les enjeux de l'information et de la communication, 15(2a), 5-19.

Bullich, V. et Guignard, T. (2014b). Les plates-formes de contenus numériques: une nouvelle intermédiation?. Dans L. Jeanpierre et O. Roueff (dir.), La culture et ses intermédiaires (p. 201-210). Paris, France: Éditions des Archives contemporaines.

Farchy, J., Méadel, C. et Anciaux, A. (2017). Une question de comportement. Recommandation des contenus audiovisuels et transformations numériques. tic\&société, 10(2-3), 168198. 
Filière de l'audiovisuel et plateformes SVOD : une analyse croisée des stratégies de Disney et Netflix

George, É. (2014). Quelles perspectives critiques pour aborder les TIC ?. tic\&société, 8(1-2), 9-29.

Guibert, G., Rebillard, F. et Rochelandet, F. (2016). Médias, culture et numérique. Approches socioéconomiques. Paris, France : Armand Colin.

Jenkins, H. (2008). Convergence Culture: Where Old and New Media Collide. New York, NY : New York University Press.

Lyubareva, I., Benghozi, P.-J. et Fidele, T. (2014). Online Business Models in Creative Industries. Diversity and Structure. International studies of management and organization, 44(4), 43-62.

Matthews, J. (2015). Passé, présent et potentiel des plateformes collaboratives. Réflexions sur la production culturelle et les dispositifs d'intermédiation numérique. Les enjeux de l'information et de la communication, 16(1), 57-71.

McCabe, J. et Akass, K. (2008). It's not TV, it's HBO's original programming: Producing quality TV. Dans M. Leverette (dir.), It's not TV: Watching HBO in the post-television era (p. 8394). New York, NY : Routledge.

Miège, B. (2004). L'économie politique de la communication. Hermès, La Revue, (38), 46-54.

Miège, B. (2007a). La Société conquise par la communication. 2. La communication entre l'industrie et l'espace public. Grenoble, France : Presses universitaires de Grenoble.

Miège, B. (2007b). La Société conquise par la communication. 3. Les Tic entre innovation technique et ancrage social. Grenoble, France : Presses universitaires de Grenoble.

Miège, B. (2017). Les industries culturelles et créatives face à l'ordre de l'information et de la communication. Grenoble, France: Presses universitaires de Grenoble.

Mosco, V. (1998). The Political Economy of Communication. Londres, Royaume-Uni : Sage Publications.

Mœglin, P. (2007). Des modèles socio-économiques en mutation. Dans P. Bouquillion et Y. Combès (dir.), Les industries de la culture et de la communication en mutation (p. 151-162). Paris, France : L'Harmattan. 


\section{Lucien PERTICOZ}

Pénard, T. et Rallet, A. (2014). De l'économie des réseaux aux services en réseaux: nouveau paradigme, nouvelles orientations. Réseaux, (184-185), 71-93.

Perticoz, L. (2012). Les industries culturelles en mutation : des modèles en question. Revue française des sciences de l'information et de la communication, (1). doi: 10.4000/rfsic.112

Schumpeter, J. (2004). Capitalisme, socialisme et démocratie. Paris, France : Payot.

Smyrnaios, N. (2017). Les GAFAM contre l'internet: une économie politique du numérique. Paris, France: INA Éditions.

Wasko, J. (2001). Understanding Disney: The Manufacture of Fantasy. Cambridge, Royaume-Uni : Polity Press.

Weygand, F. (2008). Économie de la «société de l'information » Quoi de neuf ?. tic\&société, 2(2), 59-80. 